\title{
Efficacy of Systemic Postoperative Pulmonary Rehabilitation After Lung Resection Surgery
}

\author{
Soo Koun Kim, $\mathrm{MD}^{1,2}$, Young Hyun Ahn, $\mathrm{MD}^{1,3}$, Jin A Yoon, $\mathrm{MD}^{1,2}$, Myung Jun Shin, $\mathrm{MD}^{1,2,4}$, \\ Jae Hyeok Chang, MD ${ }^{1,2}$, Jeong Su Cho, $\mathrm{MD}^{2,4,5}$, Min Ki Lee, $\mathrm{MD}^{2,4,6}$, Mi Hyun Kim, $\mathrm{MD}^{2,4,6}$, \\ Eun Young Yun, $\mathrm{PhD}^{7}$, Jong-Hwa Jeong, $\mathrm{MS}^{1}$, Yong Beom Shin, $\mathrm{MD}^{1,2,4}$
}

\begin{abstract}
${ }^{1}$ Department of Rehabilitation Medicine, Pusan National University School of Medicine, Busan; ${ }^{2}$ Biomedical Research Institute, Pusan National University Hospital, Busan; ${ }^{3}$ Department of Rehabilitation Medicine, Medwill Hospital, Busan; ${ }^{4}$ Regional Center for Respiratory Diseases, Pusan National University Hospital, Busan; ${ }^{5}$ Department of Thoracic and Cardiovascular Surgery, Pusan National University School of Medicine, Busan; ${ }^{6}$ Department of Internal Medicine, Pusan National University School of Medicine, Busan; ${ }^{7}$ Department of Biostatistics, Clinical Trial Center, Pusan National University Hospital, Busan, Korea
\end{abstract}

Objective To investigate the efficacy of systemic pulmonary rehabilitation (PR) after lung resection in patients with lung cancer.

Methods Forty-one patients undergoing lung resection were enrolled and classified into the experimental ( $\mathrm{n}=31)$ and control groups $(n=10)$. The experimental group underwent post-operative systemic PR which was conducted $30 \mathrm{~min} /$ day on every hospitalization day by an expert physical therapist. The control group received the same education about the PR exercises and were encouraged to self-exercise without supervision of the physical therapist. The PR group was taught a self-PR program and feedback was provided regularly until 6 months after surgery. We conducted pulmonary function testing (PFT) and used a visual analog scale (VAS) to evaluate pain, and the modified Borg Dyspnea Scale (mBS) to measure perceived respiratory exertion shortly before and 2 weeks, 1,3 , and 6 months after surgery.

Results A significant improvement on the VAS was observed in patients who received systemic PR $>3$ months. Significant improvements in forced vital capacity (FVC) and mBS score were observed in patients who received systemic PR >6 months ( $p<0.05)$. Other PFT results were not different compared with those in the control group.

Conclusion Patients who received lung resection suffered a significant decline in functional reserve and increases in pain and subjective dyspnea deteriorating quality of life (QoL). Systemic PR supervised by a therapist helped improve reduced pulmonary FVC and QoL and minimized discomfort during the postoperative periods in patients who underwent lung resection.

Keywords Pulmonary rehabilitation, Lung resection, Quality of life

Received May 13, 2014; Accepted October 10, 2014

Corresponding author: Yong Beom Shin

Department of Rehabilitation Medicine, Pusan National University Hospital, Pusan National University School of Medicine, 179 Gudeok-ro, Seo-gu, Busan 602-739, Korea

Tel: +82-51-240-7484, Fax: +82-51-247-7485, E-mail: yi0314@gmail.com

(c) This is an open-access article distributed under the terms of the Creative Commons Attribution Non-Commercial License (http://creativecommons.org/ licenses/by-nc/4.0) which permits unrestricted noncommercial use, distribution, and reproduction in any medium, provided the original work is properly cited. Copyright $\odot 2015$ by Korean Academy of Rehabilitation Medicine 


\section{INTRODUCTION}

Pulmonary rehabilitation (PR) treatment is frequently considered for patients with chronic obstructive pulmonary disease (COPD). PR is effective for decreasing symptoms, minimizing disease deterioration, and improving daily activities of patients with COPD [1]. The role of PR in a preoperative setting has been broadly explored in patients with pulmonary emphysema registered for lung volume resection surgery [2]. However, there is a lack of data on the value of postoperative PR in patients who have undergone lung resection surgery; thus we designed this study to investigate the effect of a systemic PR program on pulmonary function, respiratory difficulties, and quality of life (QoL) that was supervised by PR therapist after lung resection.

\section{MATERIALS AND METHODS}

\section{Patients}

A case control study with 74 patients diagnosed with lung cancer and who underwent lung resection surgery at our hospital from October to December 2010 were enrolled. Of the 74 patients, 46 accepted, and the patients who did not accept entering the PR program were considered the control group. We excluded patients who were diagnosed with a cardiovascular disease, diabetes mellitus, hypertension, and COPD. Patients with a history of radiotherapy were also excluded. Per protocol analysis testing was performed. After the per protocol analysis, 31 patients (18 men and 13 women; mean age, 60.5 \pm 11.6 years; mean number of hospitalization days, 7.0 \pm 5.4 days) who had undergone systemic PR and 10 controls (six men and four women; mean age, $64.2 \pm 5.8$ years; mean number of hospitalization days, $7.5 \pm 3.2$ days) were enrolled in this study (Table 1).

\section{Pulmonary rehabilitation program}

The PR program was comprised of range of motion exercises, chest expansion exercises, segmental breathing, and respiratory muscle training with incentive spirometry and breath-control training during aerobic activity. The PR program was started on the day of surgery unless a surgical complication occurred. However, complica-

Table 1. Baseline patient characteristics

\begin{tabular}{|c|c|c|c|}
\hline Characteristic & PR group $(n=31)$ & Control group $(n=10)$ & p-value ${ }^{a)}$ \\
\hline Age (yr) & $60.55 \pm 11.68$ & $64.20 \pm 5.79$ & 0.199 \\
\hline Gender & & & 1.000 \\
\hline Male & $18(58.1)$ & $6(60.0)$ & \\
\hline Female & $13(41.9)$ & $4(40.0)$ & \\
\hline Diagnose code & & & 0.378 \\
\hline Benign & $5(16.1)$ & $3(30.0)$ & \\
\hline Malignant extent of resection & $26(83.9)$ & $7(70.0)$ & \\
\hline VATS & & & 0.332 \\
\hline VATS & $27(87.1)$ & $7(70.0)$ & \\
\hline Thoracotomy & $4(12.9)$ & $3(30.0)$ & \\
\hline Lobectomy site & & & 0.869 \\
\hline Upper lobe & $18(58.1)$ & $5(50.0)$ & \\
\hline Middle lobe & $3(9.7)$ & $1(10.0)$ & \\
\hline Lower lobe & $10(32.2)$ & $4(40.0)$ & \\
\hline Postoperative chemotherapy & & & 0.696 \\
\hline Yes & $21(70.0)$ & $8(80.0)$ & \\
\hline No & $9(30.0)$ & $2(20.0)$ & \\
\hline Hospitalization (day) & $7.00(6.00-10.00)$ & $7.50(6.75-11.50)$ & $0.830^{\mathrm{b})}$ \\
\hline
\end{tabular}

Values are presented as mean \pm standard deviation, number (\%) or median (25th-75th percentile).

PR, pulmonary rehabilitation; VATS, video-assisted thoracoscopic surgery.

a) -test or Fisher exact test. ${ }^{\text {b) }}$ Mann-Whitney U test. 


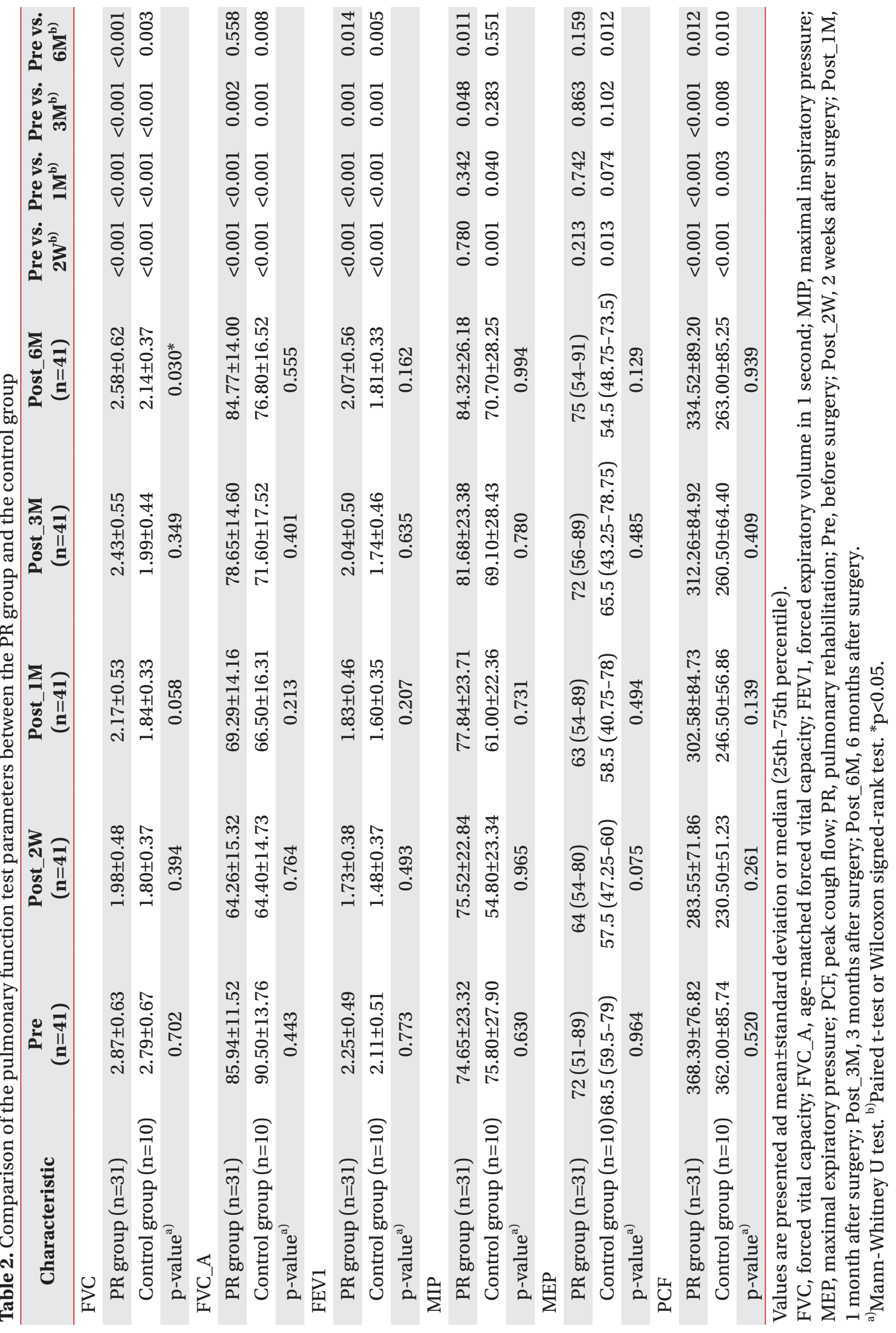


tions, including problematic bleeding, infection, pneumothorax, hemothorax, or pneumonia did not occur in our patients. Systemic PR was offered by a skilled physical therapist $30 \mathrm{~min} /$ day during all admission days, and the self-PR program education with regular feedback was offered until 6 months after. Chest expansion exercises and segmental breathing exercises were provided to avoid shrinkage of the lungs postoperatively. The chest expansion exercises worked the upper and lower extremities and rib motion of the trunk, such as pump-handle and bucket-handle motions, to increase the anterior-posterior and transverse dimensions of the rib cage during inspiration. Segmental breathing exercises consisted of surface resistance with the patient's hand on the resected lobe to expand the target lobe. For increase of Respiratory muscle training with an inspirometer was provided to increase respiratory muscle strength and volume as well as breathing control. Inspirometer training was done in a sitting position, and the target inspiration volume was $80 \%$ of the last recorded forced vital capacity (FVC) value. Training was recommended at least three times a day for at least 20 minutes each. FVC was evaluated at the follow-up when the chest tube removed to regulate training intensity and frequency. Breathing-control training consisted of education about preventing interruptions in respiration due to pain or discomfort. Aerobic exercise included indoor ambulation activities, such as stair up and down exercises. Aerobic exercise was performed at moderate intensity which regulated by using the modified Borg Dyspnea Scale (mBS) after the 6-minute walking test.

Control group patients received a PR education program and were recommended to self-exercise without supervision of a physical therapist.

\section{Assessments}

All patients underwent pulmonary function testing (PFT), a visual analog scale (VAS) for respiratory QoL, and the $\mathrm{mBS}$ to assess perceived respiratory difficulty shortly before and 2 weeks, 1, 3, and 6 months after surgery. The MicroLab ML3500 MK8 (CareFusion, Basingstoke, Hampshire, UK) was used for the PFT assessment. PFT was performed by an experienced pulmonary therapist. The evaluation was performed with the subject
(A)

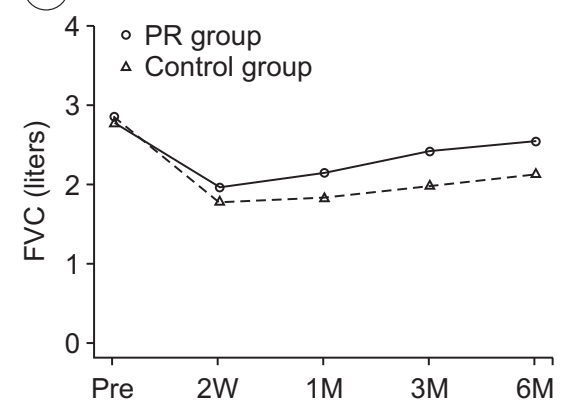

(D)

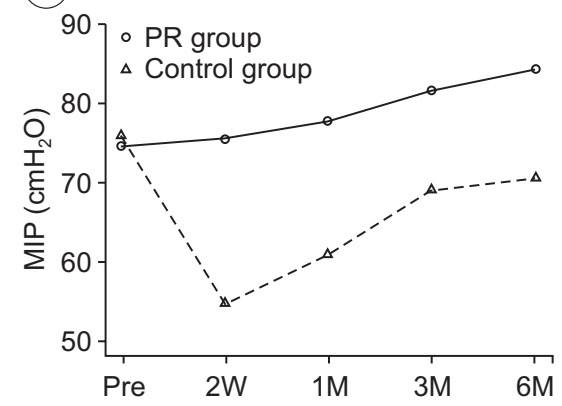

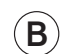

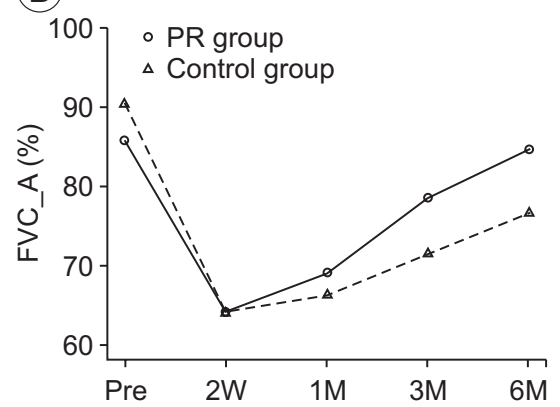

(E)

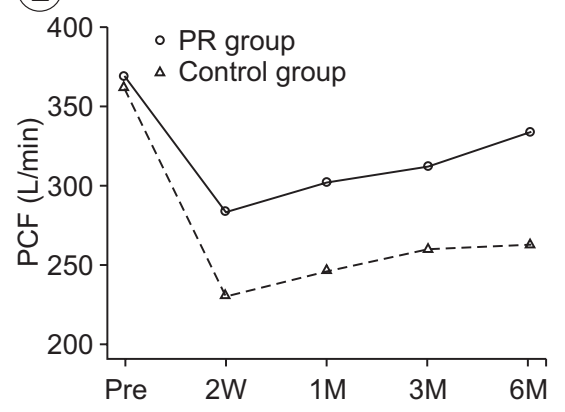

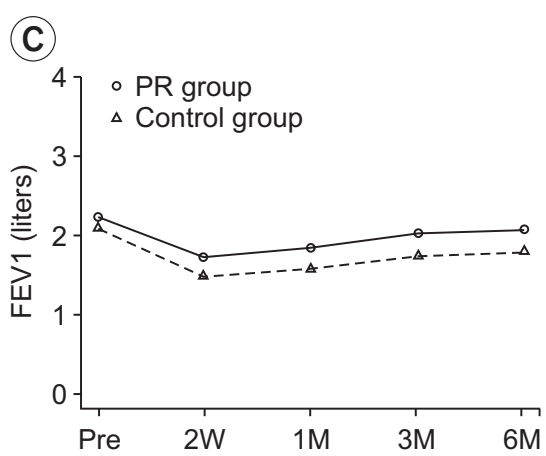

Fig. 1. Comparison of (A) FVC, (B) FVC_A, (C) FEV1, (D) MIP, and (E) PCF between the pulmonary rehabilitation (PR) group and control group before and 2 weeks and 1, 3, and 6 months after surgery. FVC, forced vital capacity; FVC_A, age-matched FVC; FEV1, forced expiratory volume in 1 second; MIP, maximal inspiratory pressure; PCF, peak cough flow. 
in a sitting position and FVC, forced expiratory volume in 1 second (FEV1), maximal inspiratory pressure (MIP), maximal expiratory pressure (MEP), and peak cough flow (PCF) were measured. Dyspnea intensity was assessed on a 0 to 10 numeric rating scale, where 0 was no symptoms and 10 was the worst possible dyspnea. The mBS was used to monitor respiratory intensity; 0 was nothing to 10 was maximum shortness of breath [3].

\section{Statistical analysis}

A comparative analysis was conducted between the control and PR groups using the t-test or Fisher's exact test for normally distributed and the Mann-Whitney U test for non-normally distributed baseline PFT, VAS, and mBS data. The Wilcoxon signed-rank test was used to compare the parameters at follow-up. Values are presented as mean \pm standard deviation, numbers (\%), or medians (25th-75th percentile). All statistical tests were conducted using SPSS software ver. 18.0 (SPSS Inc., Chicago, IL, USA).

\section{RESULTS}

PFT

The FVC and FEV1 values of the experimental and control groups decreased significantly during the postoperative follow-up assessment compared with those at the preoperative assessment. FVC 6 months after surgery in the systemic PR group improved significantly compared with that of the control group (2.58 \pm 0.62 vs. $2.14 \pm 0.37$; $\mathrm{p}=0.030$ ). Age-matched FVC (FVC_A) values in the systemic PR group were not different 6 months postoperatively compared with the preoperative values $(\mathrm{p}=0.558)$. This result indicates that systemic PR is more effective for improving some pulmonary functions. The FEV1, MIP, MEP, and PCF values tended to be improved after PR compared with those in the control group, but were not statistical significant (Table 2, Fig. 1).

\section{VAS}

The VAS evaluated 3 months after surgery showed difference between the experimental group and control group (VAS $=1.0$ [range, $0.00-2.00]$ vs. VAS $=2.5$ [range, 1.75-3.25]; $\mathrm{p}=0.014)$. The systemic PR group showed more significant improvement on the VAS compared with that of the control group 6 months after surgery (VAS $=0.0$

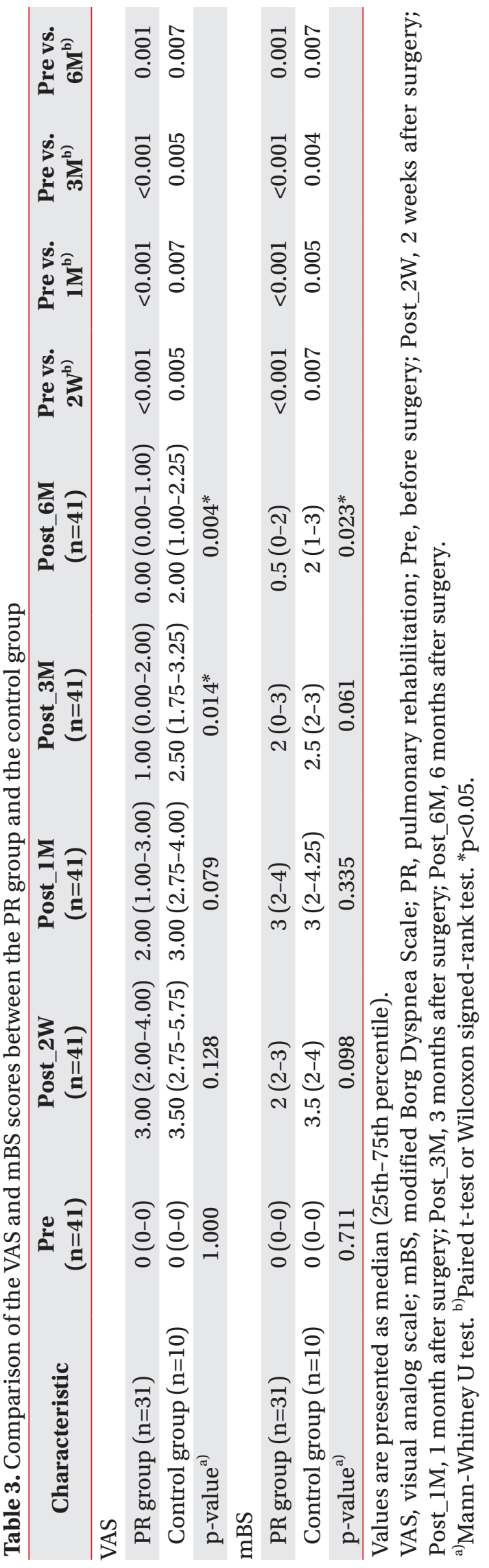


[range, $0.00-1.00$ ] vs. VAS $=2.0$ [range, $1.00-2.25] ; \mathrm{p}=0.004$ ) (Table 3, Fig. 2).

mBS

Patients who were treated with systemic PR were more comfortable with their breathing than the control group 6 months after lung resection $(\mathrm{mBS}=0.5$ [range, $0-2]$ vs. $\mathrm{mBS}=2.0$ [range, 1-3]; $\mathrm{p}=0.023$ ) (Table 3, Fig. 2).

\section{DISCUSSION}

Lobectomy is surgical resection of one or more lung lobes [4]. Lung resection is the most effective treatment for lung cancer [5]; however, pulmonary resection has a direct negative influence on pulmonary function and QoL $[2,6,7]$. We compared respiratory difficulties and chest pain, which affect QoL, and conducted PFT in patients who underwent a lung resection operation and did or did not receive systemic PR postoperatively.

Win et al. [8] investigated the consequences of lung resection on pulmonary function in patients with lung cancer. She showed that pulmonary resection produces sharp declines in FVC and FEV1 1 month after surgery, and slow recovery was observed until 3 months after lung resection. FVC and FEV1 rarely improved subsequently. Funakoshi et al. [9] also studied the long-term effects of lobectomy on patients with lung cancer. He revealed an upswing in reduced FVC and FEV1 values 12 months after surgery but no significant statistical improvement in pulmonary function was detected. Thus, we used a 6-month follow-up period to verify the effects of systemic

(A)

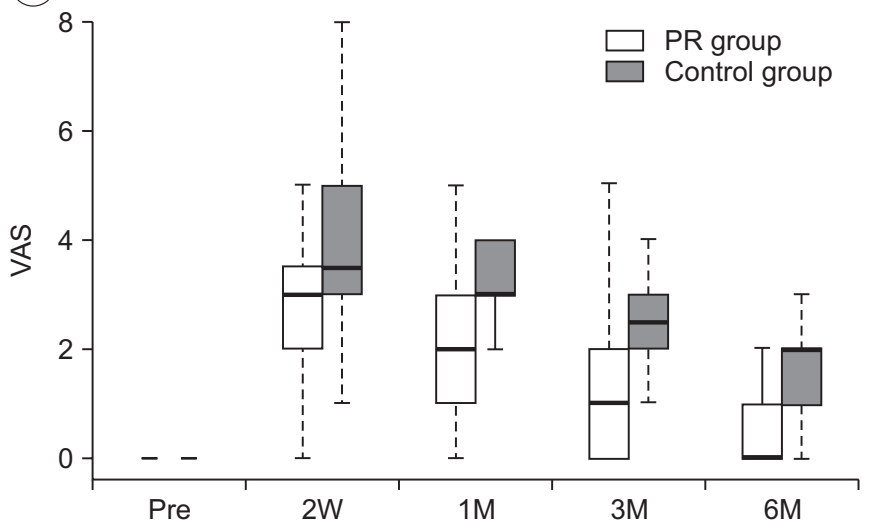

PR.

In the present study, the postoperative FVC and FEV1 values were significantly worse than their preoperative values in all patients: the patients who had undergone lung resection lost $33 \%$ of FVC and $28 \%$ of FEV1 2 weeks after surgery (Table 2). Neural depression due to pain causes a decrease in respiratory muscle power, which results in a decrease in FVC and FEV1. In addition, resected lung parenchyma is a direct cause of the decline of pulmonary function [9]. The FVC_A value in the systemic PR group 6 months after the operation was not different from the preoperative value $(\mathrm{p}=0.558)$. The FVC value of the experimental group improved significantly 6 months after the surgery $(\mathrm{p}=0.030)$, demonstrating that a 6 -month systemic PR program improved FVC more than self-exercise program and the improvement matched the preoperative level in patients who underwent lung resection.

Nomori et al. [10] showed that MIP and MEP decrease $58 \% 1$ week after a thoracotomy and then improve slowly to $88 \% 12$ weeks after lung surgery compared with preoperational values. In the present study, the experimental group showed significantly improved MIP (109\% vs. $91 \%$ ) and MEP ( $100 \%$ vs. 95\%) 12 weeks after surgery than the control group (Table 2). The systemic PR group also tended to prevent the decline of MIP ( $101 \%$ vs. $72 \%)$ compared with the control group 2 weeks after lung resection. Although the results were not significant, systemic PR tended to help recovery of respiratory muscle strength after lung resection.

Bott et al. [11] suggested strategies for assisted airway

(B)

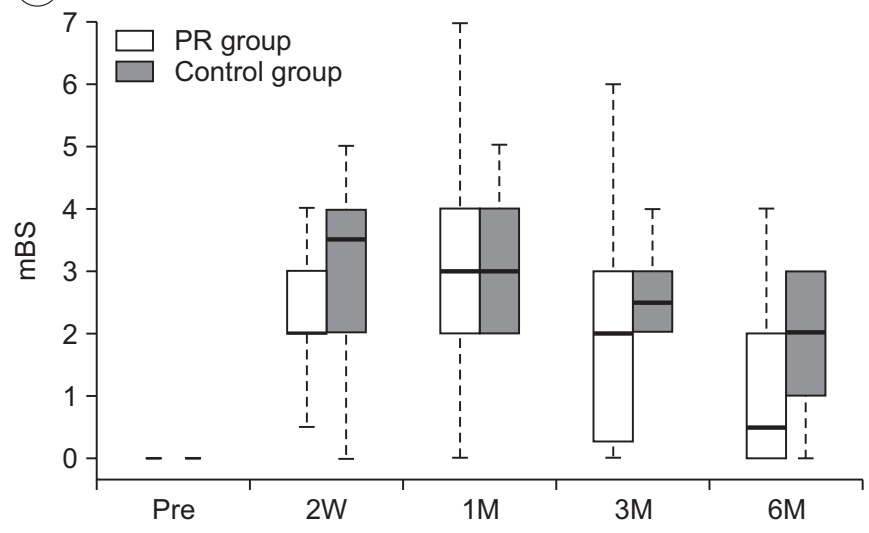

Fig. 2. Changes in (A) VAS and (B) mBS scores before and 2 weeks and 1, 3, and 6 months after surgery. VAS, visual analog scale; mBS, modified Borg Dyspnea Scale. 
clearance to increase PCF $>270 \mathrm{~L} / \mathrm{min}$ when it was $\leq 270$ $\mathrm{L} / \mathrm{min}$ in a medically stable patient. In our investigation, PCF values in the systemic PR group were always $>270 \mathrm{~L} /$ min, whereas PCF values in the control group were $<270$ $\mathrm{L} /$ min until 6 months after surgery. This result shows that systemic PR can help prevent pulmonary toileting in patients who have undergone lung resection.

In most published studies after lung resection, mental scales evaluating QoL of patients following lung resection usually remain unchanged, whereas physical scales for pain show significant impairment $[12,13]$. In this study, a VAS was used to evaluate pain, which is related to respiration and QoL. Miyoshi et al. [14] established changes in pulmonary function and exercise capacity during a 30-day postoperative period and concluded that early postoperative recovery is attributable to repair of the surgical injury to the chest wall and the resulting alleviation of pain. Pain treatment improves QoL and helps provide early recovery from surgical damage. The systemic PR had a remarkable ability to alleviate pain. 3 months after surgery. Surprisingly, the VAS score was 0 in the experimental group at the 6-month follow-up, showing the efficacy of PR to decrease pain. Continuous and regular respiratory rehabilitation enhanced patient QoL.

The mBS was used to measure respiratory difficulties and to evaluate QoL related to respiration [15]. The control group $\mathrm{mBS}$ result reflected the degree of respiratory difficulties following a pulmonary resection. Lung resection involves reducing lung volume and causes surgical injury to the chest wall. Respiratory difficulties originate from the restrictive damage to the chest wall and a decrease in diaphragm muscular activity [9]. In contrast, the respiratory difficulties of patients who received regular PR for 6 months improved after lung resection. Thus, the PR program may have helped recovery of the restrictive chest wall damage.

The systemic PR effects lasted from 3-6 months. No complications were observed during the PR program; therefore, the systemic PR program during the days patients were in the hospital could help the transition to a home-based PR program after discharge.

This study had several limitations that should be discussed. Only 10 control group patients were included because of a high dropout rate; thus, we could not apply the intention-to-treat principle. However, the per protocol set was generated sufficiently to ensure that the data would exhibit treatment effects according to the underlying scientific model. Moreover, we did not account for the amount of resected lung parenchyma. Postoperative lung volume is influenced by the location and size of the mass. The lobe of operation affected postoperative lung volume, even if the parenchyma removed, which is larger during lower lobectomy, the reduction of postoperative total lung volume is less than that resulting from an upper lobectomy [16]. No difference in the frequency of lobectomy sites was observed between the two groups (Table 1). We measured tumor size using radiological findings and no significant difference were detected between the groups ( $3.5 \mathrm{~cm}$ [range, 1.8-5.2 cm] vs. $2.5 \mathrm{~cm}$ [range, 1.4-3.2 cm]; $\mathrm{p}=0.281$ ). Therefore, the reductions in tumor size and lung volume probably did not affect the PR in this study. We could have evaluated the therapeutic influence of PR more objectively if we had compared the quantities functional lung tissue removed or diaphragm movement. Despite these limitations, we revealed the advantage of systemic PR in patients who underwent lung resection surgery.

In conclusion, more than 3 months of systemic PR improved QoL, and more than 6 months of systemic PR relieved respiratory difficulties and improved postoperative FVC.

\section{CONFLICTS OF INTEREST}

No potential conflict of interest relevant to this article was reported.

\section{ACKNOWLEGMENTS}

This study was supported by a 2-Year Research Grant from Pusan National University. And the authors would like to thank Regional Center for Respiratory Diseases of Pusan National University Hospital for their support.

\section{REFERENCES}

1. Derom E, Marchand E, Troosters T. Pulmonary rehabilitation in chronic obstructive pulmonary disease. Ann Readapt Med Phys 2007;50:615-26.

2. Bobbio A, Chetta A, Ampollini L, Primomo GL, Internullo E, Carbognani P, et al. Preoperative pulmonary rehabilitation in patients undergoing lung resection 
for non-small cell lung cancer. Eur J Cardiothorac Surg 2008;33:95-8.

3. Chetta A, Castagnaro A, Foresi A, Del Donno M, Pisi G, Malorgio R, et al. Assessment of breathlessness perception by Borg scale in asthmatic patients: reproducibility and applicability to different stimuli. J Asthma 2003;40:323-9.

4. Rochester CL. Pulmonary rehabilitation for patients who undergo lung-volume-reduction surgery or lung transplantation. Respir Care 2008;53:1196-202.

5. Pearson FG. Current status of surgical resection for lung cancer. Chest 1994;106(6 Suppl):337S-339S.

6. Lima LN, da Silva RA, Gross JL, Deheinzelin D, Negri EM. Assessment of pulmonary function and quality of life in patients submitted to pulmonary resection for cancer. J Bras Pneumol 2009;35:521-8.

7. Manzano RM, Carvalho CR, Saraiva-Romanholo BM, Vieira JE. Chest physiotherapy during immediate postoperative period among patients undergoing upper abdominal surgery: randomized clinical trial. Sao Paulo Med J 2008;126:269-73.

8. Win T, Groves AM, Ritchie AJ, Wells FC, Cafferty F, Laroche $\mathrm{CM}$. The effect of lung resection on pulmonary function and exercise capacity in lung cancer patients. Respir Care 2007;52:720-6.

9. Funakoshi Y, Takeda S, Sawabata N, Okumura Y, Maeda H. Long-term pulmonary function after lobectomy for primary lung cancer. Asian Cardiovasc Thorac Ann 2005;13:311-5.
10. Nomori H, Horio H, Fuyuno G, Kobayashi R, Yashima $\mathrm{H}$. Respiratory muscle strength after lung resection with special reference to age and procedures of thoracotomy. Eur J Cardiothorac Surg 1996;10:352-8.

11. Bott J, Blumenthal S, Buxton M, Ellum S, Falconer C, Garrod R, et al. Guidelines for the physiotherapy management of the adult, medical, spontaneously breathing patient. Thorax 2009;64 Suppl 1:i1-51.

12. Hernandez Alava M, Wailoo A, Wolfe F, Michaud K. The relationship between EQ-5D, HAQ and pain in patients with rheumatoid arthritis. Rheumatology (Oxford) 2013;52:944-50.

13. Novoa N, Varela G, Jimenez MF, Aranda JL. Influence of major pulmonary resection on postoperative daily ambulatory activity of the patients. Interact Cardiovasc Thorac Surg 2009;9:934-8.

14. Miyoshi S, Yoshimasu T, Hirai T, Hirai I, Maebeya S, Bessho T, et al. Exercise capacity of thoracotomy patients in the early postoperative period. Chest 2000;118:384-90.

15. Boshuizen RC, Vincent AD, van den Heuvel MM. Comparison of modified Borg scale and visual analog scale dyspnea scores in predicting re-intervention after drainage of malignant pleural effusion. Support Care Cancer 2013;21:3109-16.

16. Sengul AT, Sahin B, Celenk C, Basoglu A. Postoperative lung volume change depending on the resected lobe. Thorac Cardiovasc Surg 2013;61:131-7. 